\title{
Doppler shift and broadening in solar wind turbulence
}

\author{
Y. Narita ${ }^{1}$, K.-H. Glassmeier ${ }^{2,3}$, U. Motschmann ${ }^{4,5}$, and M. Wilczek ${ }^{6 *}$ \\ ${ }^{1}$ Space Research Institute, Austrian Academy of Sciences, Schmiedlstr. 6, A-8042 Graz, Austria \\ ${ }^{2}$ Institut für Geophysik und extraterrestrische Physik, Technische Universität Braunschweig, Mendelssohnstr. 3, D-38106, Germany \\ ${ }^{3}$ Max-Planck-Institut für Sonnensystemforschung, Max-Planck-Str. 2, D-37191 Katlenburg-Lindau, Germany \\ ${ }^{4}$ Institut für Theoretische Physik, Technische Universität Braunschweig, Mendelssohnstr. 3, D-38106, Germany \\ ${ }^{5}$ Deutsches Zentrum für Luft- und Raumfahrt, Institut für Planetenforschung, Rutherfordstr. 2, D-12489 Berlin, Germany \\ ${ }^{6}$ Institut für Theoretische Physik, Westfälische Wilhelms-Universität, Wilhelm-Klemm-Str. 9, D-48149 Münster, Germany
}

(Received October 31, 2012; Revised December 11, 2012; Accepted December 13, 2012; Online published January 31, 2013)

\begin{abstract}
We propose a method to evaluate the Doppler shift and broadening using magnetic field data in solar wind turbulence. A model of the energy spectrum is constructed in the wavenumber-frequency domain in the direction of the mean flow, and it is compared with that determined directly from four-point magnetic field data of Cluster spacecraft using the method of least square fitting. The model assumes the frequencies and wavelengths are low enough, and long enough, to be in the inertial range of turbulence. The comparison gives the result that the Doppler shift is consistent with that expected from the measurement of ion bulk speed, whereas the Doppler broadening is not small but far larger than the root-mean-square of ion bulk speed fluctuation possibly due to large-scale counter-propagating waves. Measurement of the Doppler shift can be used as an independent method to determine the mean flow speed, and that of Doppler broadening as a method to evaluate Taylor's frozen-in flow hypothesis. The finite Doppler broadening also implies that Taylor's hypothesis is invalid even at low frequencies in the solar wind.
\end{abstract}

Key words: Doppler shift, Doppler broadening, wavenumber-frequency spectrum, multi-spacecraft measurements.

\section{Introduction}

Since the 1960s, the solar wind has repeatedly been interpreted to be in a fully-developed turbulent state (Coleman, 1968; Matthaeus and Goldstein, 1982; Marsch and Tu, 1990; Podesta et al., 2007), as the energy spectrum in the frequency domain often exhibits a power-law with an index close to $-5 / 3$, the slope of the inertial-range spectrum for fluid turbulence (Kolmogorov, 1941). In addition, the solar wind is a supersonic and super-Alfvénic flow with the respective Mach numbers being typically 8 to 10 . For these reasons, the frozen-in flow hypothesis proposed by Taylor (1938), interpreting the measured frequencies as pure Doppler shift due to the mean flow, is thought to be a valid assumption in the study of solar wind turbulence.

Taylor's hypothesis is essentially a unique mapping from the measured frequencies into the streamwise wavenumbers, but the quality of mapping diminishes if Doppler broadening associated with large-scale random fluctuations of the flow is present in the measurement. Therefore, it is important to study how large the Doppler broadening is in the solar wind with respect to the Doppler shift, and by

\footnotetext{
* Now also at: Department of Mechanical Engineering, The Johns Hopkins University, 3400 North Charles Street, Baltimore, MD 21218, USA.

Copy right(c) The Society of Geomagnetism and Earth, Planetary and Space Sciences (SGEPSS); The Seismological Society of Japan; The Volcanological Society of Japan; The Geodetic Society of Japan; The Japanese Society for Planetary Sciences; TERRAPUB.
}

doi:10.5047/eps.2012.12.002 comparing the two effects, it is possible to validate Taylor's hypothesis. The impact of Doppler broadening on Taylor's hypothesis was discovered in studies of fluid turbulence by generalizing Taylor's hypothesis for the two-time, two-point correlation function (He and Zhang, 2006) and for the energy spectrum in the wavenumber-frequency domain (Wilczek and Narita, 2012).

Here, we propose an analysis method to evaluate the Doppler shift and broadening from magnetic field measurements in the solar wind and to validate the use of Taylor's hypothesis. Such an analysis requires both the construction of an energy spectrum model and the development of a high-resolution spectral analysis technique for multi-spacecraft data (Narita, 2012), and it has not been possible to perform the analysis before. The model used here assumes that the frequencies and wavelengths are low enough, and long enough, to be in the inertial range of solar wind turbulence. Magnetic field data from four Cluster spacecraft (Escoubet et al., 2001; Balogh et al., 2001) in the solar wind provide an excellent opportunity for this study. The presented method is useful not only in the validation of Taylor's hypothesis (scientific use) but also in estimating the flow speed in the solar wind only from magnetic field measurements (experimental use).

\section{Doppler Effect in the Energy Spectrum}

The energy spectrum model in the wavenumberfrequency domain is constructed in the presence of Doppler 
shift and broadening as follows:

$$
E_{\text {model }}(k, \omega ; U, V)=\frac{E_{0}\left|k / k_{0}\right|^{-5 / 3}}{\sqrt{2 \pi k^{2} V^{2}}} \exp \left[-\frac{(\omega-k U)^{2}}{2 k^{2} V^{2}}\right] \text {, }
$$

where $k$ and $\omega$ denote the streamwise wavenumber and the frequency in the observer's frame (in the Eulerian sense), respectively. The spectrum is characterized by two free parameters: the mean flow velocity $U$, and the random sweeping velocity $V$. They are associated with the Doppler shift $k U$ and broadening $k V$, respectively. It is a generalization of the inertial-range spectrum for fluid turbulence developed by Fung et al. (1992), Kaneda (1993), and Wilczek and Narita (2012), and is used here as the minimal and simplest form of magnetic energy spectrum for plasma turbulence. For fluid turbulence, $V$ is associated with the rootmean-square of the large-scale velocity fluctuation.

Equation (1) is a model claiming that the inertial-range spectrum is constructed on the assumption of a Gaussian character of the plasma velocity fluctuation coupled to Kolmogorov's $-5 / 3$ law on the wavenumber dependence. The model exhibits several interesting properties. First, it is analytically integrable both over frequencies and wavenumbers. The one-dimensional (1D) spectra are obtained as a power-law with the same spectral index, $-5 / 3$, in both the wavenumber and the frequency domain, and, furthermore, this property holds independently from the choice of $U$ and $V$. Second, Eq. (1) gives a more exact meaning of Taylor's hypothesis: it is recovered by taking the limit $V \rightarrow 0$ in Eq. (1) such that the fluctuation energy is constrained to the Doppler relation as the spectrum is characterized by the Dirac delta function: $E(k, \omega) \propto \delta(\omega-k U)$. By doing so, a mapping is valid from the frequencies $\omega$ into the streamwise wavenumbers by the Doppler shift $k U$. A finite Doppler broadening makes the mapping inaccurate and Taylor's hypothesis invalid.

There is no direct observation or confirmation of the Gaussian frequency distribution in space or laboratory plasma turbulence, but indirect evidence is the fact that Eq. (1) predicts the same power-law index for both wavenumber and frequency spectra. The 1D frequency spectrum for solar wind turbulence is known to be well fitted by the power-law with the index $-5 / 3$, and this observational fact motivates the use of our spectral model. We assume no time evolution for the small-scale turbulent field itself. Matthaeus et al. (2010) argue, based on Eulerian time correlation, that Taylor's hypothesis begins to be inaccurate beyond above 3 hours, which gives a constraint in our analysis method concerning the time-length of the measurement.

\section{Application to Solar Wind Turbulence 3.1 Observation}

The energy spectrum model is applied to turbulent fluctuations of the interplanetary magnetic field measured by the four Cluster spacecraft. They encountered the interplanetary space ahead of the Earth's bow shock on February 20, 2002, from 1930 to 2000 UT. We use the magnetic field data and ion data from Cluster in the interplanetary space obtained by the fluxgate magnetometer FGM (Balogh et al., 2001) and the electrostatic analyzer CIS-HIA (Rème

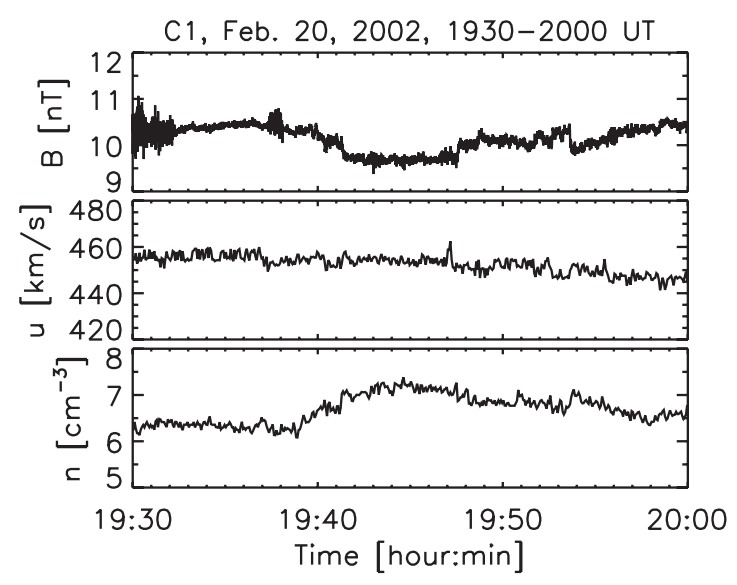

Fig. 1. Time series plot of magnetic field magnitude, ion bulk speed, and ion number density measured by Cluster- 1 spacecraft in the interplanetary space (ahead of the Earth bow shock).

et al., 2001), respectively. Cluster formed a nearly regular tetrahedron with the inter-spacecraft separation about $100 \mathrm{~km}$. Figure 1 displays the magnetic field magnitude, the ion bulk speed, and the ion number density recorded on board the Cluster-1 spacecraft. The mean fields, in particular the ion bulk speed, may be regarded as nearly constant: magnetic field magnitude $\langle B\rangle=10.1 \mathrm{nT}$, ion bulk velocity $\langle u\rangle=443.0 \mathrm{~km} / \mathrm{s}$, and ion density $\langle n\rangle=6.3 \mathrm{~cm}^{-3}$. The root-mean-square of the ion bulk speed fluctuation is only moderate, $\sqrt{|\delta u|^{2}}=6.8 \mathrm{~km} / \mathrm{s}$. The time interval contains no ion foreshock activity (which appears as a low-frequency disturbance) but that of electrons occasionally, as electric field data exhibit a high-frequency disturbance $(2-80 \mathrm{kHz})$ from the WHISPER instrument (Décréau et al., 2001).

The wavenumber-frequency spectrum, is determined from the measurement of four-point magnetic field at Cluster, and the spectrum and is used to estimate the parameters $U$ and $V$ by comparison with the spectrum model. In this way, we construct the best-fitted spectrum model for the analyzed time interval. Transformation of the time series data into the frequency domain is performed by a Fast Fourier Transform, and that from the spatial coordinate into wavevectors is performed by the Multi-point Signal Resonator (MSR) technique developed particularly for the analysis of four-point magnetic field data from Cluster (Narita et al., 2011). MSR enables direct determination of the energy spectrum as done by Narita et al. (2010) and Sahraoui et al. (2010). In contrast to their earlier methods, MSR is a very-high-resolution adaptive filter technique, and is constructed as a fusion of the minimum variance projector (the wave telescope technique, see Motschmann et al., 1996; Glassmeier et al., 2001) with the MUSIC algorithm (Multiple Signal Classification) developed by Schmidt (1986) based on the analysis of eigenvalues and eigenvectors of the measured covariance matrix. We determine the fourdimensional (4D) energy spectrum $E_{\text {obs }}(\vec{k}, \omega)$ first, and then obtain the two-dimensional (2D) spectrum $E_{\text {obs }}(k, \omega)$ in the flow direction by averaging the 4D spectrum over wavenumbers perpendicular to the flow. The ion data are used for the orientation of wavenumber. 
(a) Measured spectrum

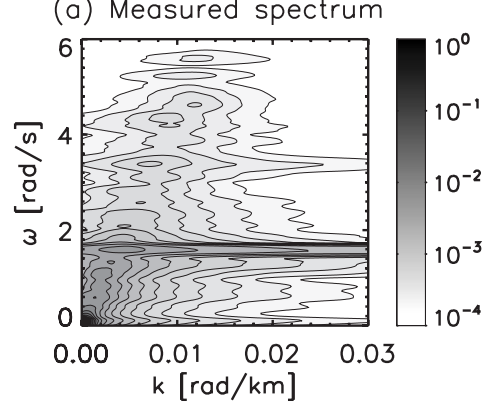

(b) Fitted spectrum

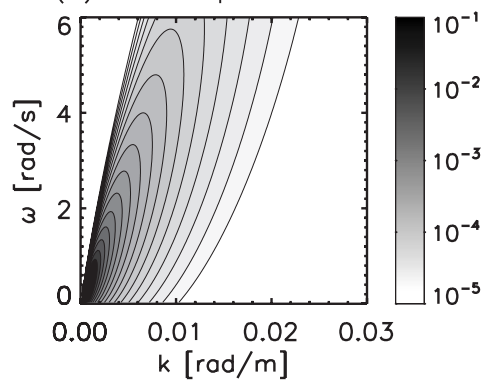

Fig. 2. Energy spectra in the wavenumber-frequency domain spanned by wavenumber along the flow and frequency in the spacecraft frame. Panel (a) is the spectrum derived from magnetic field data of Cluster, in the time intervals shown in Fig. 1, using the high-resolution wavenumber analysis technique MSR. The panel (b) shows the best-fitted spectrum for the parameter set determined by the method of least square estimator (cf. Fig. 3). Contour levels are given in units of $\mathrm{nT}^{2} /[(\mathrm{rad} / \mathrm{s})(\mathrm{rad} / \mathrm{km})]$.

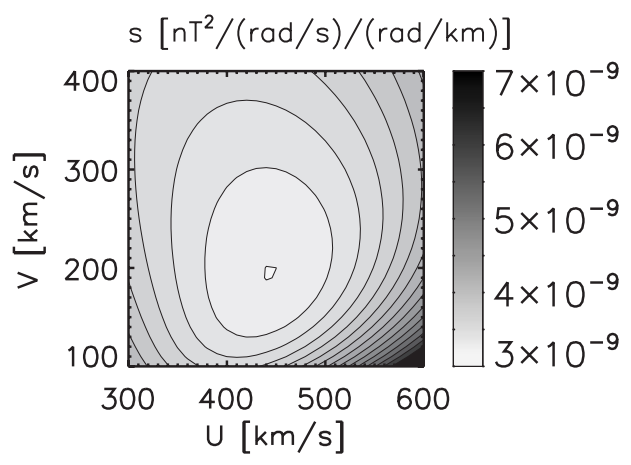

Fig. 3. Deviation $s$ of the model spectrum from the measured one in the parameter space spanned by the mean velocity $U$ and the random sweeping velocity $V$.

Figure 2 displays the measured wavenumber-frequency spectrum using the MSR technique (panel (a)) and the bestfitted model obtained from the method of least square fitting (panel (b), explained below). The coordinate of the spectrum is spanned by the wavenumber in the direction of the ion bulk flow and the frequency in the spacecraft frame. The measured spectrum exhibits maximum values at the smallest frequencies and wavenumbers, and the spectral energy decays toward higher frequencies and wavenumbers, while it shows two extended directions with enhanced energy in the wavenumber-frequency diagram: one is oblique from the frequency and wavenumber axes (which is fitted as the Doppler shift); and the other is parallel to the wavenumber axis. The latter represents spacecraft spin at a frequency about $1.6 \mathrm{rad} / \mathrm{s}$ (cf. spin period at about 4 seconds) and its second harmonics due to spin-coupled disturbances at the spacecraft. It is an artifact of the measurement, and is not used in the analysis.

\subsection{Least square fitting}

The optimal values of the two parameters, $U$ and $V$, are obtained by least square fitting under the following conditions: frequency less than the spin frequency $(\omega<$ $2 \pi \times 0.25 \mathrm{rad} / \mathrm{s}$ ); wavenumber larger than the cutoffwavenumber $(k \geq 0.005 \mathrm{rad} / \mathrm{km})$. The energy level $E_{0}$ is adapted to the value at lower cutoff wavenumber. In the least square fitting, the deviation is investigated between the measured and model spectra. The deviation $s$ is averaged over wavenumbers and frequencies, and is defined as

$$
\begin{aligned}
s(U, V)= & \frac{1}{N_{k} N_{\omega}}\left[\sum_{i=1}^{N_{k}} \sum_{j=1}^{N_{\omega}} \mid E_{\text {model }}\left(k_{i}, \omega_{j} ; U, V\right)\right. \\
& \left.-\left.E_{\mathrm{obs}}\left(k_{i}, \omega_{j}\right)\right|^{2}\right]^{1 / 2}
\end{aligned}
$$

where and $N_{k}$ and $N_{\omega}$ are the number of data points in wavenumbers and frequencies (200 points in each domain). The result of the fitting is displayed in Fig. 3. It is a contour plot of the deviation $s$ in the parameter space spanned by the mean velocity $U$ and the random sweeping velocity $V$. The smallest deviation is found with the combination of $U=443.5 \mathrm{~km} / \mathrm{s}$ and $V=194.9 \mathrm{~km} / \mathrm{s}$. These parameters are used to produce the fitted spectrum in Fig. 2. The mean velocity $U$ agrees with that obtained from the ion measurements (cf. time series plot in Fig. 1), $\langle u\rangle=443.0 \mathrm{~km} / \mathrm{s}$, but the random sweeping velocity $V$ is markedly large and cannot be explained simply by the root-mean-square of the ion velocity fluctuation (at about $7 \mathrm{~km} / \mathrm{s}$ ). The large random sweeping velocity implies that the use of Taylor's hypothesis cannot be justified in solar wind turbulence even at low frequencies.

\section{Summary and Discussion}

Multi-point measurements of the magnetic field are a useful tool to investigate plasma turbulence properties in the interplanetary space, as they have the potential to determine the mean flow velocity as well as the random sweeping velocity by comparing the measured wavenumber-frequency spectrum with the theoretical model in Eq. (1). We obtain from the fitting procedure that the mean flow velocity (characterized by the parameter $U$ ) is in a good agreement with the directly-measured ion bulk velocity $\langle u\rangle$. The capability of evaluation of the mean flow velocity using multipoint magnetic field data should be emphasized here. This method is valid as long as the observed plasma is in a fullydeveloped turbulent state such as in the solar wind, and provides an independent method of flow velocity measurement other than direct ones. For future multi-spacecraft missions, the presented method can be used as an additional means of calibration for plasma experiments on board. We also find that the Doppler broadening is not small but far larger than expected from the ion measurement. The random sweeping velocity is of the same order as the mean flow velocity. In the analyzed time interval the ratio $V / U$ is about 0.44 . Tay- 
lor's frozen-in flow hypothesis is therefore not a very valid assumption, at least in the analyzed time interval of solar wind turbulence. It should be interpreted in such a way that the frequency spectrum is Kolmogorov's power-law, not because Taylor's hypothesis is valid but because it is one of the properties of the inertial-range spectrum as modeled in Eq. (1).

The spectrum model used here is based on the hydrodynamic treatment of the inertial-range spectrum in the sense that it neglects the magnetohydrodynamic effects of such as large-scale Alfvén waves. In order to correctly obtain the Doppler shifted spectrum from that in the plasma frame, it is necessary to take into account the spectral information of both the wavenumber space and the frequency space. Namely, the Lorentz transformation should be performed for both the frequency and the wavenumber spaces. In other words, in the limit of $U=0$, the model equation (1) should be written as a function of the wavenumber and the frequency that represents the dispersion relation for magnetohydrodynamics. If large-scale Alfvén waves were present with the propagation speed $V_{\mathrm{ph}}=V_{\mathrm{A}} \cos \theta$ (where $V_{\mathrm{A}}$ is the Alfvén velocity and $\theta$ is the propagation angle from the mean magnetic field), the spectral peak should split from a single Doppler relation line $\omega=k U$ into two branches: $\omega=k\left(U+V_{\mathrm{ph}}\right)$ and $\omega=k\left(U-V_{\mathrm{ph}}\right)$ due to the large-scale waves propagating forward and backward with respect to the flow. Therefore, we interpret our result such that a splitting might exist in the measured spectrum; splitting is so weak that it is recognized as a large frequency broadening. In this scenario, the sweeping velocity $V$ is expected to be about the doubled Alfvén speed, and, in fact, the ratio $V / V_{\mathrm{A}}$ is about 2.3 in the analyzed interval (the sweeping velocity at about $195 \mathrm{~km} / \mathrm{s}$ from the fitting; Alfvén velocity at about $85 \mathrm{~km} / \mathrm{s}$, estimated from the magnetic field strength and the ion number density).

The spatial scale of the large-scale fluctuation, either eddies or Alfvén waves, cannot be estimated in the present analysis alone. Different approaches are, however, possible to estimate the spatial scale associated with the sweeping velocity: deriving information on large-scale structures from the frequency spectrum; extending the model spectrum to include the energy-containing range in the wavenumber domain as in von Kármán's model; Cluster data analysis for large-scale tetrahedron phase; mapping coronal structure scale onto the Cluster orbit (nearly at 1 AU). The combination of the two different studies, one for analysis of random sweeping velocity and the other for analysis of energy-containing eddy scales, would give more complete information on the driving mechanism of turbulence in the interplanetary space.

Acknowledgments. Ion data from the Cluster CIS HIA instrument team and Cluster Active Archive are acknowledged. This work is financially supported by the Bundesministerium für Wirtschaft und Technologie and the Deutsches Zentrum für Luftund Raumfahrt, Germany, under contract 50 OC 0901, and Collaborative Research Center 963, "Astrophysical Flow, Instabilities, and Turbulence" of the German Science Foundation.

\section{References}

Balogh, A., C. M. Carr, M. H. Acuña, M. W. Dunlop, T. J. Beek, P.
Brown, K.-H. Fornaçon, E. Georgescu, K.-H. Glassmeier, J. Harris, G. Musmann, T. Oddy, and K. Schwingenschuh, The Cluster magnetic field investigation: overview of in-flight performance and initial results, Ann. Geophys., 19, 1207-1217, 2001.

Coleman, P. J., Jr., Turbulence, viscosity, and dissipation in the solar-wind plasma, Astrophys. J., 153, 371, 1968.

Décréau, P. M. E., P. Fergeau, V. Krasnoselskikh, E. Le Guirriec, M Lévêque, Ph. Martin, O. Randriamboarison, J. L. Rauch, F. X. Sené, H. C. Séran, J. G. Trotignon, P. Canu, N. Cornilleau, H. de Féraudy, H. Alleyne, K. Yearby, P. B. Mögensen, G. Gustafsson, M. André, D. C. Gurnett, F. Darrouzet, J. Lemaire, C. C. Harvey, P. Travnicek, and Whisper Experimenters group, Early results from the Whisper instrument on Cluster: an overview, Ann. Geophys., 19, 1241-1258, 2001.

Escoubet, C. P., M. Fehringer, and M. Goldstein, The Cluster mission, Ann Geophys., 19, 1197-1200, 2001

Fung, J. C. H., J. C. R. Hunt, N. A. Malik, and R. J. Perking, Kinematic simulation of homogeneous turbulence by unsteady random Fourier modes, J. Fluid Mech., 236, 281-318, 1992.

Glassmeier, K.-H., U. Motschmann, M. Dunlop, A. Balogh, M. H. Acuña, C. Carr, G. Musmann, K.-H. Fornaçon, K. Schweda, J. Vogt, E. Georgescu, and S. Buchert, Cluster as a wave telescope-first results from the fluxgate magnetometer, Ann. Geophys., 19, 1439-1447, 2001 (correction 21, 1071, 2003).

He, G.-W. and J.-B. Zhang, Elliptic model for space-time correlation in turbulent shear flows, Phys. Rev. E, 73, 055303R, 2006.

Kaneda, Y., Lagrangian and Eulerian time correlations in turbulence, Phys. Fluids A, 5, 2835-2845, 1993.

Kolmogorov, A. N., The local structure of turbulence in incompressible viscous fluid for very large Reynolds number, Dokl. Akad. Nauk. SSSR, 30, 299-303, 1941 (reprinted in Proc. R. Soc. A, 434, 9-13, 1991).

Marsch, E. and C.-Y. Tu, On the radial evolution of MHD turbulence in the inner heliosphere, J. Geophys. Res., 95, 8211-8229, 1990.

Matthaeus, W. H. and M. L. Goldstein, Measurement of the rugged invariants of magnetohydrodynamic turbulence in the solar wind, J. Geophys. Res., 87, 6011-6028, 1982.

Matthaeus, W. H., S. Dasso, J. M. Weygand, M. G. Kivelson, and K. T. Osman, Eulerian decorrelation of fluctuations in the interplanetary magnetic field, Astrophys. J. Lett., 721, L10-L13, doi:10.1088/20418205/721/1/L10, 2010.

Motschmann, U., T. I. Woodward, K. H. Glassmeier, D. J. Southwood, and J. L. Pinçon, Wavelength and direction filtering by magnetic measurements at satellite arrays: Generalized minimum variance analysis, J. Geophys. Res., 101, 4961-4966, 1996.

Narita, Y., Plasma Turbulence in the Solar System, 102 pp, Springer Briefs in Physics, Springer, Heidelberg, 2012.

Narita, Y., F. Sahraoui, M. L. Goldstein, and K.-H. Glassmeier, Magnetic energy distribution in the four-dimensional frequency and wave vector domain in the solar wind, J. Geophys. Res., 115, A04101, doi:10.1029/2009JA014742, 2010.

Narita, Y., K.-H. Glassmeier, and U. Motschmann, High-resolution wave number spectrum using multi-point measurements in space - The MultiPoint Signal Resonator (MSR) technique, Ann. Geophys., 29, 351-360, 2011.

Podesta, J. J., D. A. Roberts, and M. L. Goldstein, Spectral exponents of kinetic and magnetic energy spectra in solar wind turbulence, Astrophys. $J .$, 664, 543-548, doi:10.1086/519211, 2007.

Rème, H., C. Aoustin, J. M. Bosqued, I. Dandouras et al., First multispacecraft ion measurements in and near the Earth's magnetosphere with the identical Cluster ion spectrometry (CIS) experiment, Ann. Geophys., 19, 1303-1354, 2001.

Sahraoui, F., M. L. Goldstein, G. Belmont, P. Canu, and L. Rezeau, Three dimensional anisotropic $\mathrm{k}$ spectra of turbulence at subproton scales in the solar wind, Phys. Rev. Lett., 105, 131101, doi:10.1103/PhysRevLett.105.131101, 2010.

Schmidt, R. O., Multiple emitter location and signal parameter estimation, IEEE Trans. Ant. Prop., AP-34, 276-280, 1986.

Taylor, G. I., The spectrum of turbulence, Proc. R. Soc. Lond. A, 164, 476490, 1938.

Wilczek, M. and Y. Narita, Wave-number-frequency spectrum for turbulence from a random sweeping hypothesis with mean flow, Phys. Rev. E, 86, 066308, doi:10.1103/PhysRevE.86.066308, 2012.

Y. Narita (e-mail: yasuhito.narita@oeaw.ac.at), K.-H. Glassmeier, U. Motschmann, and M. Wilczek 